\title{
Analysis and verification of the HMGB1 signaling pathway
}

\author{
Haijun Gong ${ }^{1 *}$, Paolo Zuliani ${ }^{1 *}$, Anvesh Komuravelli ${ }^{1}$, James R Faeder ${ }^{2}$, Edmund M Clarke ${ }^{1}$ \\ From Asia Pacific Bioinformatics Network (APBioNet) Ninth International Conference on Bioinformatics \\ (InCoB2010) \\ Tokyo, Japan. 26-28 September 2010
}

\begin{abstract}
Background: Recent studies have found that overexpression of the High-mobility group box-1 (HMGB1) protein, in conjunction with its receptors for advanced glycation end products (RAGEs) and toll-like receptors (TLRs), is associated with proliferation of various cancer types, including that of the breast and pancreatic.

Results: We have developed a rule-based model of crosstalk between the HMGB1 signaling pathway and other key cancer signaling pathways. The model has been simulated using both ordinary differential equations (ODEs) and discrete stochastic simulation. We have applied an automated verification technique, Statistical Model Checking, to validate interesting temporal properties of our model.

Conclusions: Our simulations show that, if HMGB1 is overexpressed, then the oncoproteins CyclinD/E, which regulate cell proliferation, are overexpressed, while tumor suppressor proteins that regulate cell apoptosis (programmed cell death), such as p53, are repressed. Discrete, stochastic simulations show that p53 and MDM2 oscillations continue even after 10 hours, as observed by experiments. This property is not exhibited by the deterministic ODE simulation, for the chosen parameters. Moreover, the models also predict that mutations of RAS, ARF and P21 in the context of HMGB1 signaling can influence the cancer cell's fate - apoptosis or survival through the crosstalk of different pathways.
\end{abstract}

\section{Background}

The cell cycle is strictly regulated and controlled by a complex network of signaling pathways [1], comprised of hundreds of proteins. If some important proteins are mutated or there are defects in the signaling mechanisms, normal cell growth regulation will break down, possibly leading to the occurrence of cancer in the future. Moreover, a number of extracellular proteins can bind to their receptors and activate signaling pathways that promote the proliferation of cancer cells.

The high-mobility group box-1 (HMGB1) protein is a DNA-binding nuclear protein, released actively in response to cytokine stimulation, or passively during cell death [2], and it is present in almost all eukaryotic cells

\footnotetext{
* Correspondence: haijung@cs.cmu.edu; pzuliani@cs.cmu.edu

${ }^{1}$ Computer Science Department, Carnegie Mellon University, Pittsburgh, PA 15213, USA

Full list of author information is available at the end of the article
}

[3-6]. HMGB1 can activate a series of signaling components, including mitogen-activated protein kinases (MAPKs) and AKT, which play an important role in tumor growth and inflammation, through binding to different surface receptors, such as RAGE and TLR2/4. Several studies have shown that elevated expression of HMGB1 occurs in many tumors [7-10] and accelerates cell-cycle progression. Recent in vitro studies with pancreatic cancer cells [11] revealed that the targeted knockout or inhibition of HMGB1 and RAGE could increase apoptosis and suppress pancreatic cancer cell growth. This phenomenon has been also observed with lung cancer and other types of cancer cells $[8,12]$.

The HMGB1 signal transduction can influence the cell's fate by two important processes - apoptosis and cell proliferation - which are regulated respectively by the proteins p53 and CyclinE, acting in two different signaling pathways. The protein p53 is one of the most 
important tumor suppressor proteins: its activation can lead to cell cycle arrest, DNA repair, or apoptosis. Mutations of p53 occur at a frequency of $50 \%$ or higher in many different cancer types [13]. CyclinE is a cell cycle regulatory protein which regulates the G1-S phase transition during cell proliferation. Cancer cells often exhibit high expression levels of CyclinE and aberrant CyclinE activity [14]. Many studies have found evidence of crosstalk between the two signaling pathways involving p53 and CyclinE [15]. The crosstalk is regulated by tumor suppressor proteins, including ARF, P21 and FBXW7, which are also frequently mutated in many cancers. In this paper, we ask the following questions: How do these proteins and their mutations change the cell's fate - apoptosis or survival - when HMGB1 signal transduction is activated? Which signaling pathways are fundamental for describing HMGB1 signal transduction, and what mechanisms are responsible to explain recent results linking overexpression of HMGB1 with decrease of apoptosis (and increased cancer cell survival)?

To the best of the authors' knowledge, no computational model has been proposed to investigate the importance of HMGB1 in tumor proliferation. In this work, we construct a simple model of HMGB1 signal transduction to investigate tumorigenesis on the basis of known signaling pathway studies [16-21]. We also constructed a crosstalk network between these known pathways based on hypothetical mechanisms suggested by recent experiments. The HMGB1 pathway is not well understood at the mechanistic level, so our model can provide some insights into the study of HMGB1's roles in tumor proliferation. A series of deterministic and stochastic simulation experiments was conducted to investigate the properties of the HMGB1 pathway.

Finally, we analyze our pathway model against interesting behavorial properties by means of Model Checking techniques. Model Checking is an automated verification technique for hardware and software systems [22]. Recently, there has been growing interest in formal verification of stochastic systems, and, which has recently seen a growing number of applications to biological systems [23-25], by means of Model Checking techniques. The Methods section introduces statistical model checking, which we then apply to validate our pathway model against experimental results from the literature.

\section{Methods}

\section{HMGB1 signaling pathway}

Our HMGB1 signaling pathway model is illustrated in Fig. 1. It includes 31 molecular species (6 tumor suppressor proteins), 59 chemical reactions, and three different signaling pathways activated by HMGB1: the RAS-ERK, Rb-E2F and p53-MDM2 pathways. Since the interaction between HMGB1 and its receptors TLR and RAGE is not clear at the mechanistic level, RAGE is used to represent all the receptors in our model in order to reduce the number of unknown parameters. We now briefly discuss the three pathways and their crosstalk. We denote activation (or promotion) by $\rightarrow$, while inhibition (or repression) is denoted by - .

The p53-MDM2 pathway is regulated by a negative feedback loop [26]: PI3K $\rightarrow$ PIP3 $\rightarrow$ AKT $\rightarrow$ MDM2 $\dashv$ p53 $\rightarrow$ MDM2, and a positive feedback loop: p53 $\rightarrow$ $\mathrm{PTEN} \dashv \mathrm{PIP} 3 \rightarrow \mathrm{AKT} \rightarrow \mathrm{MDM} 2 \dashv \mathrm{p} 53$. The protein PI3K is activated by the toll-like receptors (TLR2/4) within several minutes after TLR2/4 activation by HMGB1 [27]. In turn, PI3K phosphorylates the phosphatidylinositol 4,5-bisphosphate (PIP2) to phosphatidylinositol $(3,4,5)$-trisphosphate (PIP3), leading to phosphorylation of AKT. The unphosphorylated oncoprotein MDM2, which is one of p53's transcription targets [28], resides in the cytoplasm, and cannot enter the nucleus until it is phosphorylated by activated AKT. The phosphorylated MDM2 translocates into the nucleus to bind with p53, inhibiting p53's transcription activity and initializing p53 polyubiquitination [29], which targets it for degradation. Also, p53 can regulate the transcription of PTEN [30], a tumor suppressor protein, which can hydrolyze PIP3 to PIP2, thereby inhibiting the activation of AKT and MDM2.

The RAS-ERK pathway is the activation sequence: RAS $\rightarrow$ RAF $\rightarrow$ MEK $\rightarrow$ ERK $\rightarrow$ CyclinD. Activation of RAGE by HMGB1 leads to RAS activation, which in turn activates its effector protein RAF. Activated RAF will phosphorylate the MEK proteins (mitogen-activated protein kinase kinases (MAPKK)), leading to the phosphorylation of ERK1/2 (also called MAPKs). Activated ERK can phosphorylate some transcription factors which activate the expression of the regulatory protein CyclinD and Myc, enabling progression of the cell cycle through the G1 phase. K-RAS, a member of the RAS protein family, is found to be mutated in over $90 \%$ of pancreatic cancers [31].

The $R b-E 2 F$ pathway is composed of the interactions: CyclinD $\dashv \mathrm{Rb} \dashv \mathrm{E} 2 \mathrm{~F} \rightarrow$ CyclinE $\dashv \mathrm{Rb}$. The Rb-E2F pathway regulates the G1-S phase transition in the cell cycle during cell proliferation. E2F is a transcription factor that can activate the transcription of many proteins involved in DNA replication and cell-cycle progression [32]. In quiescent cells, E2F is bound by unphosphorylated $\mathrm{Rb}$ - a tumor suppressor protein - forming an RbE2F complex which inhibits E2F's transcription activity. E2F will be activated and released when its inhibitor $\mathrm{Rb}$ is phosphorylated by some oncoproteins (CyclinD and Myc in Fig. 1), leading to the transcription of CyclinE and Cyclin-dependent protein kinase 2 (CDK2) which promote cell-cycle progression. CyclinE, in turn, 


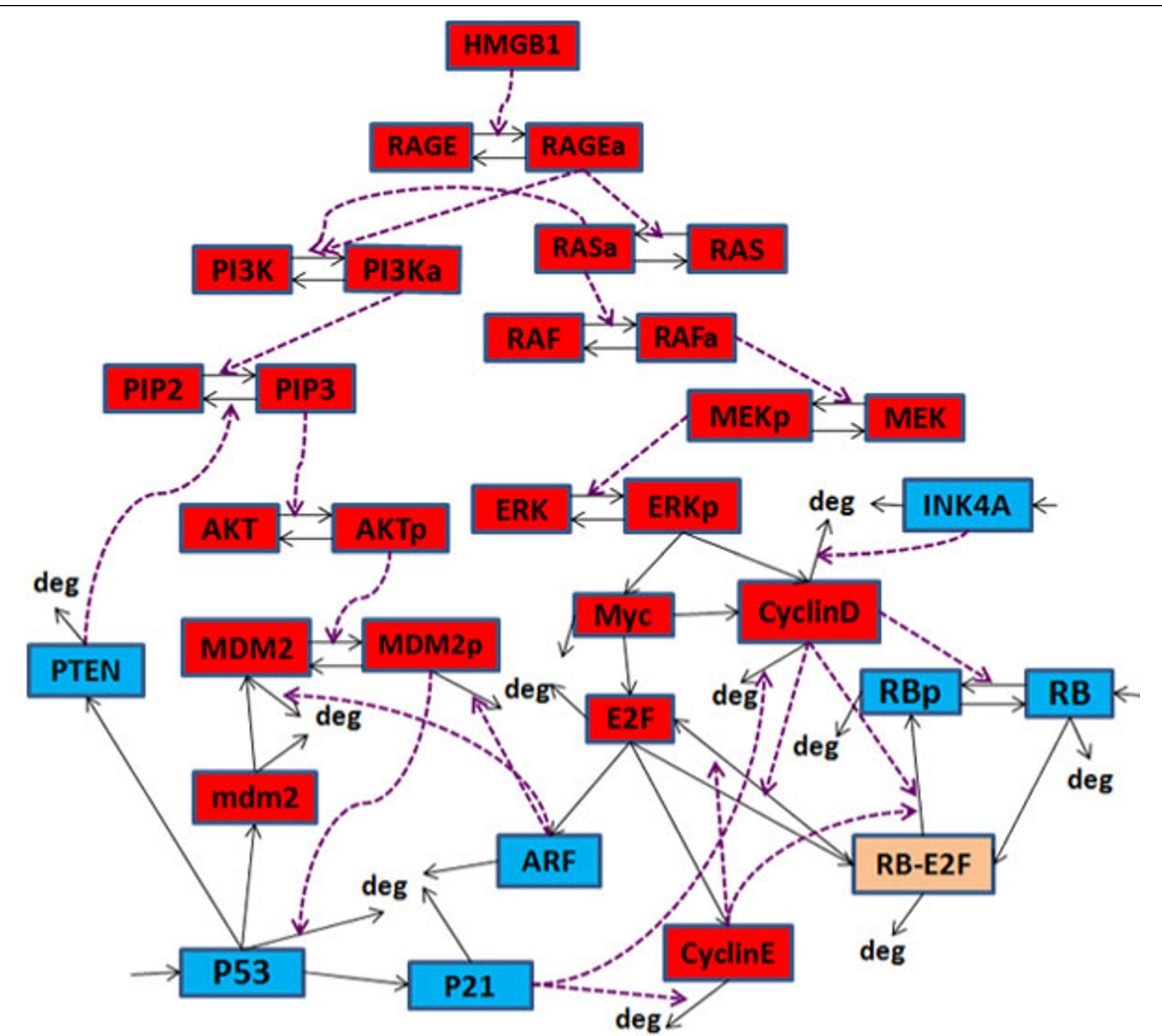

Figure 1 Schematic view of HMGB1 signal transduction. Blue nodes represent tumor suppressor proteins; red nodes represent oncoproteins/ lipids; brown node represents protein complex formed by oncoprotein E2F and tumor suppressor protein RB. Solid lines with arrows denote protein transcription, degradation or changes of molecular species; dashed lines with arrows denote activation processes.

continues to inhibit the activity of $\mathrm{Rb}$, leading to a positive feedback loop [33-35]. Fig. 1 shows that the activity of CyclinD-CDK4/6 (only CyclinD is shown in Fig. 1) is inhibited by the tumor suppressor protein INK4A, which is inactivated in up to $90 \%$ pancreatic cancers [36].

The crosstalk between these pathways can influence the cell's fate since the three signaling pathways in HMGB1 signal transduction are not independent. As shown in Fig. 1, the oncoprotein RAS can also activate the PI3K-AKT signaling pathway; the tumor suppressor ARF protein induced by E2F can bind to MDM2 to promote its rapid degradation and stabilize p53. Furthermore, it has been experimentally observed [13] that the p53-dependent tumor suppressor proteins P21 and FBXW7 can inhibit the activity of cyclin dependent kinases (In Fig. 1, we use P21 to represent both P21 and FBXW7's contribution). Mutations of RAS, ARF, P21 and FBXW7 have been found in many cancers $[31,36,37]$. One of our aims is to investigate how these mutations might influence the cell's fate.

In the HMGB1 model, all substrates are expressed in the number of molecules; proteins with the subscript " $a$ " or " $p$ " correspond respectively to active or phosphorylated forms of the proteins. For example,

- RAGE (RAGE $)_{a}$ - inactive (active) form of HMGB1's receptor 
- MDM2 (MDM2 $\left.2_{p}\right)$ - unphosphorylated (phosphorylated) MDM2.

We denote the mRNA transcript of MDM2 by $m d m 2$. We assume that the total number of active and inactive forms of the RAGE, PI3K, PIP, AKT, RAS, RAF, MEK, and ERK molecules is constant. For example, AKT + $\mathrm{AKT}_{p}=\mathrm{AKT}_{\text {tot }}, \mathrm{PIP} 2+\mathrm{PIP} 3=\mathrm{PIP}_{\text {tot }}$. We sometimes use $\mathrm{CD}$ to stand for the CyclinD-CDK4/6 complex, $\mathrm{CE}$ for CyclinE, and RE for the Rb-E2F complex.

The p53-MDM2 and RAS-ERK pathways have been studied individually using deterministic ODE methods [16-19,32]. We instead formulated a reaction model corresponding to the reactions illustrated in Fig. 1 in the form of rules specified in the BioNetGen language [38]. We used Hill functions to describe the rate laws governing protein synthesis, including PTEN, MDM2, CyclinD (CD), Myc, E2F and CyclinE (CE). Our choice was motivated by several studies [19,39-41], which showed that transcription rates of these proteins are sigmoidal functions of transcription factor (TF) concentrations with positive cooperative Hill coefficients. We used mass action rules for other types of chemical reactions. Both ODEs and Gillespie's stochastic simulation algorithm (SSA) [42] are used to simulate the model with BioNetGen [38]. Stochastic simulation is important because when the number of molecules involved in the reactions is small, stochasticity and discretization effects become more prominent [43-45]. In the online Additional file 1, we list 23 ordinary differential equations which describe the deterministic HMGB1 model and all the input parameters. The BioNetGen code which implements SSA and ODE models is available at [46].

Since our understanding of many chemical reactions at the mechanistic level is not clear, a large number of parameters involved in these reactions are difficult to estimate based on existing data. We emphasize that in our HMGB1 model the values for some undetermined parameters were chosen in order to produce a qualitative agreement with previous experiments.

\section{Model Checking}

Model Checking $[22,47]$ is one of the leading techniques for the automated verification and analysis of hardware and software systems. Given a high-level behavior specification, a model checker verifies whether a system (or model) satisfies it. A specification might be satisfied by many different models. Thus, model checking is the process of determining whether or not a given system model satisfies (is a model of) a property describing the desired behavior of the system. Mathematically, system models take the form of state-transition diagrams, while some version of temporal logic [48] is used to describe the desired properties (specifications) of system executions. A typical property stated in temporal logic is $\mathbf{G}$ (grant_req $\rightarrow \mathbf{F}$ ack), meaning that it is always $(\mathbf{G}=$ globally) true that a grant request eventually $(\mathbf{F}=$ future $)$ triggers an acknowledgment. One important aspect of Model Checking is that it can be performed algorithmically - user intervention is limited to providing a system model and a property to check.

The Probabilistic Model Checking problem (PMC) is to decide whether a stochastic model satisfies a temporal logic property with a probability greater than or equal to a certain threshold. To express temporal properties, we use a logic in which the temporal operators are equipped with bounds. For example, the property "CyclinD will always stay below 10 in the next fifty time units" is written as $\mathbf{G}^{50}(C y c l i n D<10)$. We now ask whether our stochastic system $M$ satisfies that formula with a probability greater than or equal to a fixed threshold (say 0.9), and we write $\mathrm{M} \mid=\operatorname{Pr}_{\geq 0.9}\left[\mathbf{G}^{50}(C y c l i n D<10)\right]$. In the next section, we formally define the temporal logic used in this work, Bounded Linear Temporal Logic [23].

\section{Bounded Linear Temporal Logic (BLTL)}

Let $S V$ be a finite set of real-valued variables, an atomic proposition $A P$ be a boolean predicate of the form $e_{1} \sim$ $e_{2}$, where $e_{1}$ and $e_{2}$ are arithmethic expressions over variables in $S V$, and $\sim$ is either $\geq, \leq,<,>$, or $=$. A BLTL property is built over atomic propositions using boolean connectives and bounded temporal operators. The syntax of the logic is the following:

$$
\phi::=A P\left|\phi_{1} \vee \phi_{2}\right| \phi_{1} \wedge \phi_{2}\left|\neg \phi_{1}\right| \phi_{1} \mathbf{U}^{t} \phi_{2} .
$$

The bounded until operator $\phi_{1} \mathbf{U}^{t} \phi_{2}$ requires that, within time $t, \phi_{2}$ will be true and $\phi_{1}$ will hold until then. Bounded versions of the $\mathbf{F}$ and $\mathbf{G}$ operators can be easily defined: $\mathbf{F}^{t} \phi=$ true $\mathbf{U}^{t} \phi$ requires $\phi$ to hold true within time $\left.t ; \mathrm{G}^{t} \phi=\neg \mathbf{F}^{t}\right\urcorner \phi$ requires $\phi$ to hold true up to time $t$.

The semantics of BLTL is defined with respect to traces (or executions) of a system. In our case, a trace will be the output of a simulation of a BioNetGen stochastic model. Formally, a trace is a sequence of timestamped state transitions of the form $\sigma=\left(s_{0}, t_{0}\right),\left(s_{1}\right.$, $\left.t_{1}\right), \ldots$, which means that the system moved to state $s_{i+1}$ after having sojourned for time $t_{i}$ in state $s_{i}$. The fact that a trace $\sigma$ satisfies the BLTL property $\phi$ is written as $\sigma \mid=\phi$. We denote the trace suffix starting at step $k$ by $\sigma^{k}$. We have the following semantics of BLTL:

- $\sigma^{k} \vDash A P$ if and only if $A P$ holds true in state $s_{k}$; - $\sigma^{k} \vDash \phi_{1} \wedge \phi_{2}$ if and only if $\sigma^{k} \vDash \phi_{1}$ and $\sigma^{k} \vDash \phi_{2}$;

- $\sigma^{k} \vDash \phi_{1} \vee \phi_{2}$ if and only if $\sigma^{k} \vDash \phi_{1}$ or $\sigma^{k} \vDash \phi_{2}$;

- $\sigma^{k} \vDash \neg \phi_{1}$ if and only if $\sigma^{k} \vDash \phi_{1}$ does not hold; 
- $\sigma^{k} \vDash \phi_{1} \mathbf{U}^{\mathbf{t}} \phi_{2}$ if and only if there exists $i \in N$ such that, (a) $\Sigma_{0} \leq l<i t_{k+1} \leq t$, (b) $\sigma^{k+i} \vDash \phi_{2}$ and (c) for each $0 \leq j<i, \sigma^{k+j} \vDash \phi_{1}$.

The semantics of BLTL are defined over infinite traces, but it can be shown that traces of an appropriate (finite) length are sufficient to decide BLTL properties [49].

\section{Statistical Model Checking}

We briefly explain Statistical Model Checking [50,51], the technique we use for verifying BioNetGen models simulated by Gillespie's algorithm. Statistical Model Checking treats the Probabilistic Model Checking problem as a statistical inference problem, and solves it by randomized sampling of the traces (simulations) from the model. In particular, the PMC problem is naturally phrased as a hypothesis testing problem, i.e., deciding between two hypotheses $-M \vDash \operatorname{Pr}_{\geq \theta}[\phi]$ versus $M \vDash P r_{<\theta}[\phi]$. In other words, to determine whether a stochastic system $M$ satisfies $\phi$ with a probability $p \geq \theta$, we test the hypothesis $H_{0}$ : $p \geq \theta$ against $H_{1}: p<\theta$. Sampled traces are model checked individually to determine whether a given property $\phi$ holds, and the number of satisfying traces is used by a hypothesis testing procedure to decide between $H_{0}$ and $H_{1}$. Note that Statistical Model Checking cannot guarantee a correct answer to the PMC problem. However, the probability of giving a wrong answer can be made arbitrarily small.

We have introduced a Bayesian sequential hypothesis testing approach and applied it to the verification of rulebased models of signaling pathways and other stochastic systems $[23,49]$. Sequential sampling means that the number of sampled traces is not fixed a priori, but is instead determined at "run-time", depending on the evidence gathered by the samples seen so far. This often leads to a significantly smaller number of sampled traces.

Suppose that the stochastic system $M$ satisfies the BLTL formula $\phi$ with some (unknown) probability $p$. The key idea behind statistical model checking [50] is that the behavior of $M$ (with respect to property $\phi$ ) can be modeled by a Bernoulli random variable with success parameter $p$. Such a random variable can be repeatedly evaluated via system simulation in the following way. Let $\sigma$ be a trace of $M$, then the Bernoulli random variable $X$ with (conditional) probability mass function:

$$
f(x \mid p)=p^{x}(1-p)^{1-x} \quad x \in\{0,1\}
$$

denotes the outcome of $\sigma \vDash \phi$ (i.e., model checking $\phi$ on $\sigma$ ). In other words, we have that:

$$
X= \begin{cases}1 \text { with probability } p & (\sigma \mid=\phi), \\ 0 \text { with probability } 1-p & (\sigma \mid=\neg \phi) .\end{cases}
$$

Therefore, by running a system simulation (i.e., a BioNetGen stochastic simulation) and by checking $\phi$ on the resulting trace we can obtain a sample from random variable $X$. When a sample of $X$ evaluates to 1 we call it a success, otherwise, a failure.

Recall that in hypothesis testing we decide between a null hypothesis $H_{0}$ and an alternative hypothesis $H_{1}$ :

$$
H_{0}: p \geqslant \theta \quad H_{1}: p<\theta .
$$

The Bayesian approach assumes that $p$ is given by a random variable whose distribution is called the prior distribution. The prior is usually based on our previous experiences and knowledge about the system.

Since $p$ is a probability, we need prior distributions defined over $[0,1]$. In particular, Beta priors are mathematically convenient to use. They are defined by the following probability density:

$$
\forall u \in[0,1] \quad g(u, \alpha, \beta)=\frac{1}{B(\alpha, \beta)} u^{\alpha-1}(1-u)^{\beta-1}
$$

where the Beta function $B(\alpha, \beta)$ is defined as:

$$
B(\alpha, \beta)=\int_{0}^{1} t^{\alpha-1}(1-t)^{\beta-1} d t .
$$

For later use, the Beta distribution function $F_{(\alpha ; \beta)}(u)$ of parameters $\alpha, \beta$ is defined as for all $u \in[0,1]$ as:

$$
\begin{aligned}
& F_{(\alpha, \beta)}(u)=\int_{0}^{u} g(t, \alpha, \beta) d t \\
& =\frac{1}{B(\alpha, \beta)} \int_{0}^{u} t^{\alpha-1}(1-t)^{\beta-1} d t .
\end{aligned}
$$

Let $d=\left(x_{1}, \ldots, x_{n}\right)$ denote $n$ samples of the Bernoulli random variable $X$ defined by (2). Let $H_{0}$ and $H_{1}$ be the hypotheses in (3), and suppose that the prior probabilities $P\left(H_{0}\right)$ and $P\left(H_{1}\right)$ are strictly positive and satisfy $P$ $\left(H_{0}\right)+P\left(H_{1}\right)=1$. By Bayes's theorem, the posterior probabilities of $H_{0}$ and $H_{1}$, with respect to data $d$, are:

$$
P\left(H_{i} \mid d\right)=\frac{P\left(d \mid H_{i}\right) P\left(H_{i}\right)}{P(d)} \quad(i=0,1)
$$

for every $d$ with $P(d)>0$. In our case, $P(d)$ is always non-zero (there are no impossible finite sequences of data). The hypothesis test method is based on the Bayes Factor, that is, the likelihood ratio of the sampled data with respect to the two hypotheses. The Bayes Factor $\mathscr{B}$ of sample $d$ and hypotheses $H_{0}$ and $H_{1}$ is 


$$
\mathcal{B}=\frac{P\left(d \mid H_{0}\right)}{P\left(d \mid H_{1}\right)}
$$

and by Bayes' theorem, we have that:

$$
\mathcal{B}=\frac{P\left(H_{0} \mid d\right)}{P\left(H_{1} \mid d\right)} \cdot \frac{P\left(H_{1}\right)}{P\left(H_{0}\right)} .
$$

Therefore, $B$ can be interpreted as a measure of evidence (given by the data $d$ ) in favor of $H_{0}$. Now, fix a threshold $T>1$. The algorithm iteratively draws independent and identically distributed (iid) sample traces in the form of BioNetGen stochastic simulations, and checks whether they satisfy $\phi$ (Note that BioNetGen ensures by construction that each simulation, or trace, is actually iid.) After each trace, the algorithm computes the Bayes Factor $B$ to check if it has obtained conclusive evidence. The algorithm accepts $H_{0}$ if $B>T$, and rejects $H_{0}$ (accepting $\left.H_{1}\right)$ if $\mathcal{B}<\frac{1}{T}$. Otherwise $\left(\frac{1}{T} \leq \mathcal{B} \leq T\right)$, it continues drawing iid samples. The statistical Model Checking algorithm is shown in Figure 2.

The following Proposition shows that, in our special case of Bernoulli samples, the computation of the Bayes Factor is straightforward.

Proposition 1. [49] The Bayes Factor of $H_{0}: p \geq \theta$ vs. $H_{1}: p<\theta$ with Bernoulli samples $\left(x_{1}, \ldots, x_{n}\right)$ and Beta prior of parameters $\alpha, \beta$ is:

$$
\mathcal{B}_{n}=\frac{P\left(H_{1}\right)}{P\left(H_{0}\right)}\left\{\frac{1}{F_{(x+\alpha, n-x+\beta)}(\theta)}-1\right)
$$

where $x=\sum_{i=1}^{n} x_{i}$ is the number of successes in $\left(x_{1}, \ldots\right.$, $\left.x_{n}\right)$ and $F_{(s, t)}(\cdot)$ is the Beta distribution function of parameters $s, t$.

The Beta distribution function can be efficiently computed by standard software packages. Thus, no numerical integration is required for the evaluation of the Bayes Factor.

Finally, we must show that the error probability of our decision procedure, i.e., the probability that we reject (accept) the null hypothesis although it is true (false), can be bounded.

Theorem. [49] The error probability for the sequential Bayesian hypothesis testing algorithm is bounded above by $\frac{1}{T}$ where $T$ is the Bayes Factor threshold given as input.

\section{Results and discussion}

We first conducted a series of deterministic and stochastic simulation experiments to study the properties of our HMGB1 signaling pathway model. Then, we applied the statistical model checking technique to validate some important temporal properties of our HMGB1 model.

\section{Simulation results}

We carried out a baseline simulation for four important proteins - p53, MDM2 $2_{p}$, CyclinD/E - using ODE and stochastic simulation. We set the initial value for the number of HMGB1 molecules to be $10^{3}$; Table 1 lists all proteins with nonzero initial values; the unlisted proteins are set to 0 initially.

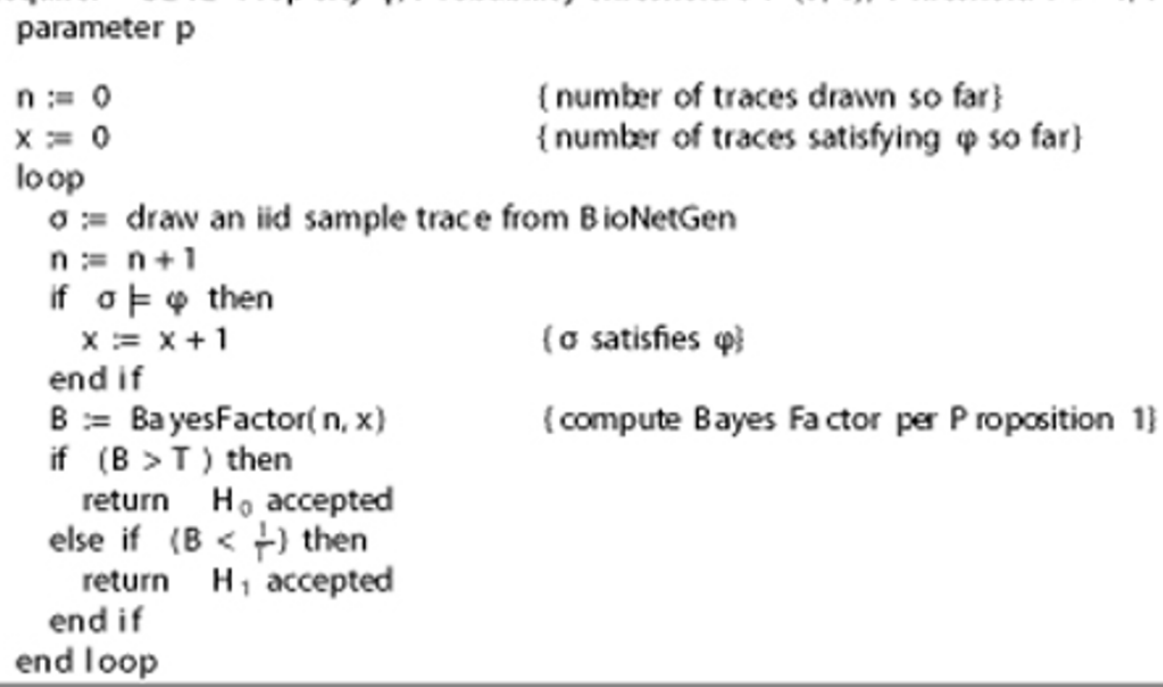

Figure 2 Statistical Model Checking Algorithm. The algorithm for Statistical Model Checking is based on Bayesian Hypothesis Testing. 
Table 1 Initial values for the model

\begin{tabular}{llllllllllll}
\hline RAGE & PI3K & PIP2 & AKT & MDM2 & MDM2 $_{\boldsymbol{p}}$ & P53 & RAS & RAF & MEK & ERK & RE \\
\hline $10^{3}$ & $10^{5}$ & $10^{5}$ & $10^{5}$ & $10^{4}$ & $2 \times 10^{4}$ & $2 \times 10^{4}$ & $10^{4}$ & $10^{4}$ & $10^{4}$ & $10^{4}$ & $10^{5}$ \\
\hline
\end{tabular}

Non-zero initial values of the proteins are listed in the table; other unlisted proteins are initially set to 0.

The baseline stochastic simulations in Fig. 3A demonstrate that the expression levels of $\mathrm{p} 53$ and $\mathrm{MDM} 2_{p}$ oscillate even after 10 hours, when the cell enters the $S$ phase (recall that cells usually remain in phase G1 for about 10 hours before moving to the $\mathrm{S}$ phase). However, oscillations are strongly damped in the ODE simulations (Fig. 3C) when the cell proceeds to the $\mathrm{S}$ phase, approximately after 10 hours. The stochastic simulation model is thus more consistent with the experimental results of Geva-Zatorsky et al. [52]. In that experiment the authors measured the dynamics of $\mathrm{p} 53$ and $\mathrm{MDM}_{2}$ in human breast cancer cells damaged by $\gamma$ radiation. It was observed that the oscillations of p53 and MDM $2_{p}$ expression levels can last more than 72 hours after irradiation.

Fig. 3B and 3D show that the CyclinE protein, which regulates the G1-S phase transition in the cell cycle, reaches its maximum at about 10 hours, after which the cell proceeds with DNA replication (S phase). How does the expression level of HMGB1 and other proteins influence the cell's fate? We varied the levels of HMGB1 and AKT to determine how they affect cell behavior. A number of studies have found that HMGB1 is overexpressed in many cancers, and the overexpression of HMGB1 and its receptors can promote cancer cell proliferation and decrease apoptosis [8,9]. In Fig. 4A-B, we increase the initial values of HMGB1 from 1 to $10^{6}$ and measure p53's maximum expression level in phase G1. We then measure the oncoproteins E2F and CyclinD/E's expression levels at 10 hours, which corresponds to the G1-S phase transition point. For the stochastic simulation, the experiment is repeated 10 times per value to compute the mean and standard errors. Fig. $4(\mathrm{~A}, \mathrm{D})$ demonstrates that the increase of HMGB1's initial value will lead to a decrease of p53's expression level, but when the number of HMGB1 molecules is over $10^{5}$, p53 will not continue to decrease. This is because HMGB1 can also activate and increase the expression level of its downstream protein E2F (Fig. 4 $(\mathrm{A}, \mathrm{D})$ ), whose overexpression will activate the transcription of the tumor suppressor protein ARF, which can inhibit MDM2's activity to stabilize p53's level. However, ARF is found to be mutated in up to $80 \%$ of pancreatic cancers $[36,53]$. This means that ARF cannot inhibit the activity of the oncoprotein MDM2, thereby leading to lower levels of the tumor suppressor p53.

Fig. 4(B, E) shows that the cell cycle regulatory proteins CyclinD/E will increase with the elevated expression of HMGB1, a behavior which could be verified by future experiments. Fig. 4(A-B, D-E) explains the

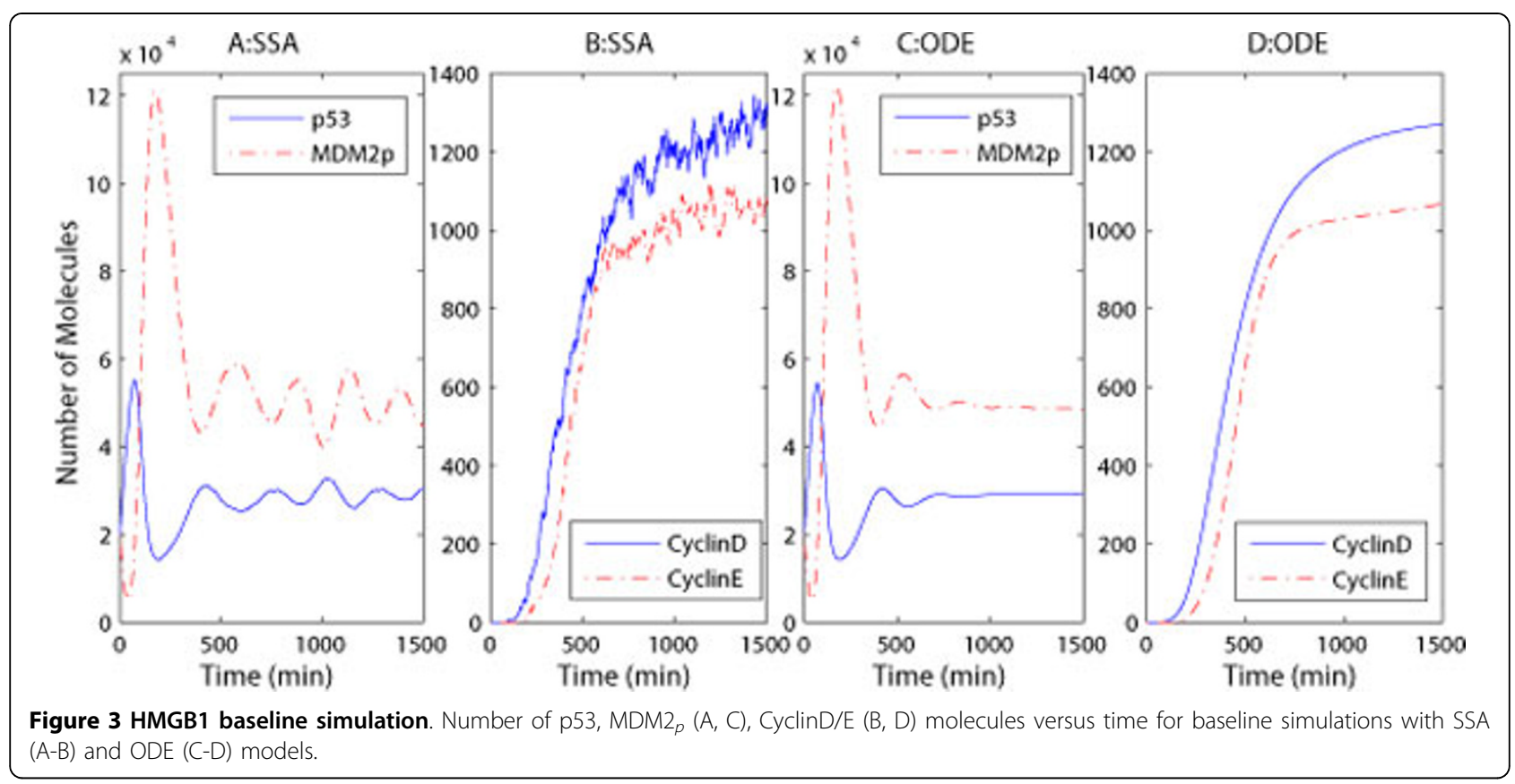




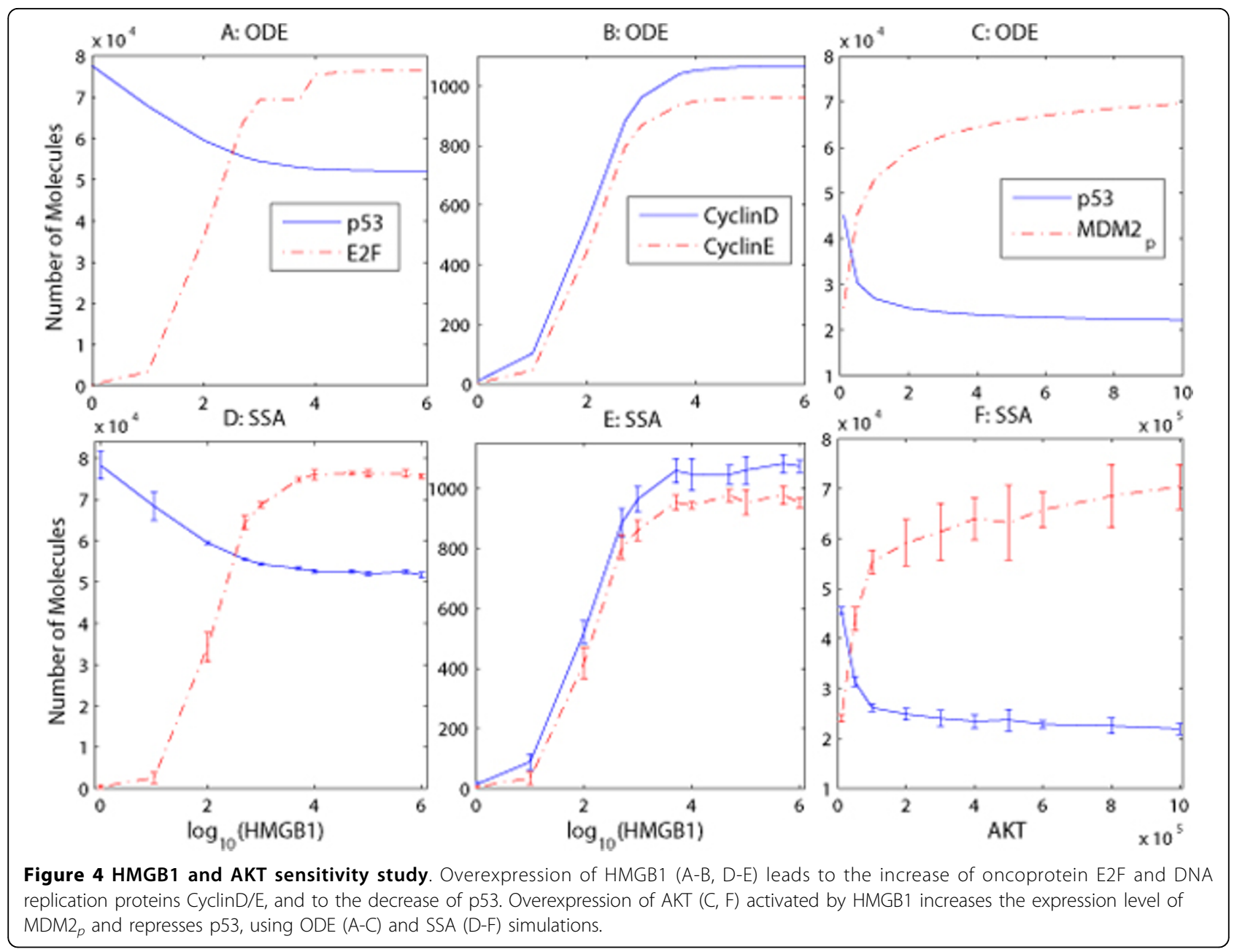

experimental discovery that the overexpression of HMGB1 decreases apoptosis and promotes DNA replication and proliferation in cancer cells.

The oncoprotein AKT is overexpressed in many cancers [54]. In Fig. 4(C, F), we first increase the number of unphosphorylated AKT molecules and fix the other proteins' concentration, then measure $\mathrm{p} 53$ and $\mathrm{MDM}_{2}$ 's expression levels at 10 hours in phase G1 after HMGB1 activates its receptor RAGE. Fig. $4(\mathrm{C}, \mathrm{F})$ shows that with the increase of AKT's expression level, p53 is repressed due to the ubiquitination initiated by the overexpressed $\mathrm{MDM} 22_{p}$, which is promoted by the activated and overexpressed AKT protein. The results in Fig. 4(C, F) suggest a way to inhibit tumor cell proliferation and induce tumor cell apoptosis through the inhibition of protein phosporylation events downstream from AKT kinases in the PI3K/AKT pathway, using an AKT kinase inhibitor (such as the drug GSK-690693 [55]).

K-RAS is a member of the RAS protein family. K-RAS mutation and ARF loss occur in more than $80 \%$ of pancreatic cancers $[36,53]$. The P21 and FBXW7 proteins are also frequently mutated in many cancers [37]. ARF and P21 play an important role in the crosstalk between the p53 and Rb pathways. ARF is able to reroute cells with oncogenic damage to p53-dependent fates through binding to MDM2 and targeting its degradation. The p53-dependent tumor suppressor proteins P21 and FBXW7 can inhibit CyclinD/E's activity to prevent the proliferation of cancer cells.

Fig. 5 shows how mutations of ARF, P21 and FBXW7, and K-RAS influence tumor suppressor and cell cycle regulatory protein levels at 10 hours in the HMGB1 signaling pathway. We use the MDM2 degradation rate driven by ARF, $d_{A R F}$ ( $d_{7}^{\prime}$ in the ODE model), to describe ARF mutations. Also, we use the Cyclin degradation rate driven by P21 ( $d_{P 21}$ for stochastic simulation, and $b_{6}^{\prime}$ for ODE simulation) to describe P21 and FBXW7 mutations. Large $d_{A R F}$ and $d_{P 21}$ values correspond to small mutations of ARF and P21 respectively, while small $d_{A R F}$ and $d_{P 21}$ values correspond to large ARF and P21 mutations in the cell. 


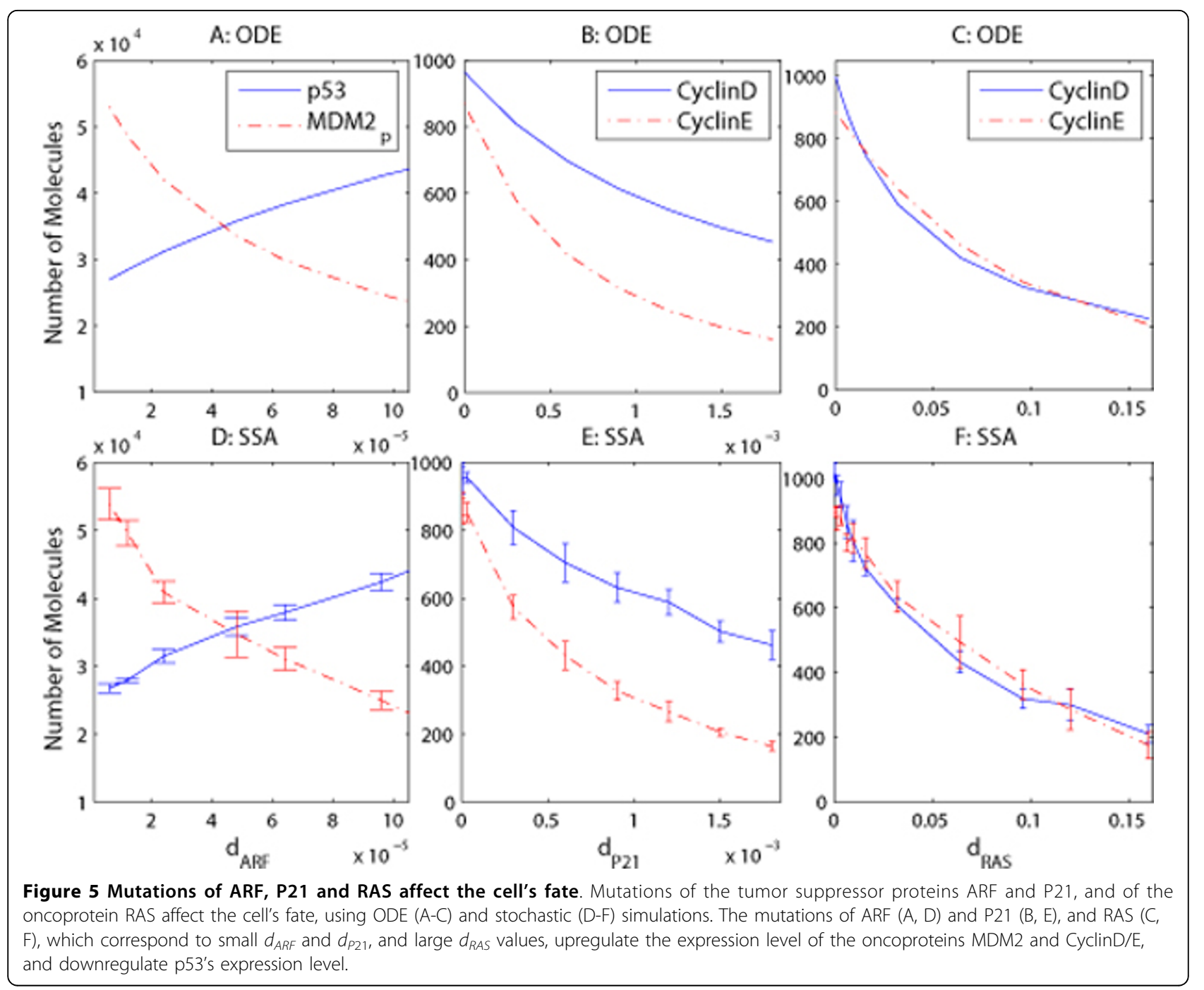

Fig. 5(A, D) shows that wild-type ARF (large $d_{A R F}$ ) can decrease the number of MDM $2_{p}$ molecules and increase p53's expression level to initiate apoptosis even if the cell proceeds to the $\mathrm{S}$ phase. Moreover, mutated ARF (smaller $d_{A R F}$ ) can not stabilize p53 expression and prevent the proliferation of cancer cells if HMGB1 is overexpressed. This could explain the phenomenon that ARF loss exists in over $80 \%$ of pancreatic cancers [36]. Fig. 5(B, E) demonstrates that CyclinD/E proteins will increase if P21 is mutated (smaller $d_{P 21}$ ), thereby accelerating cell cycle progression.

K-RAS is mutated in most cancers, especially in pancreatic cancer [31]. The activation of RAS is initiated by HMGB1 and its receptors, and the wild-type RAS can be deactivated by some kinases. Studies have found that the mutated K-RAS can not be deactivated [56], even if HMGB1 is knocked out, so it will continuously activate the downstream signaling pathways which promote cell proliferation. Fig. 5(C, F) shows that with the increase of
RAS deactivation rate $d_{R A S}\left(b_{1}\right.$ in the ODE model), the synthesis of CyclinD/E will be inhibited, but a small deactivation rate of RAS will lead to overexpression of CyclinD/E. The results visualized in Fig. 5 suggest some ways to inhibit cancer cell proliferation through inhibition or deactivation of the signaling pathways involving RAS, Cyclin, and Cyclin-dependent kinases (CDK). Recently, CDK and RAS inhibitor drugs [57-59] have been developed to inhibit tumor growth.

\section{Verification of the HMGB1 model}

We use Statistical Model Checking (SMC) to verify some fundamental properties that our model should satisfy. We test whether the model satisfies a given BLTL property with probability $p \geq 0.9$. We set the threshold $T=1000$ for the verification, so the probability of a wrong answer is smaller than $10^{-3}$.

Property 1: p53 is normally expressed at low levels in human cells. We verified the following property 
Table 2 Verification of property 1

\begin{tabular}{lllll}
\hline \multicolumn{5}{c}{ Property 1: $\boldsymbol{P r}_{\geq \mathbf{0 . 9}}\left[\mathrm{F}^{\mathbf{t}}\left(\mathrm{G}^{\mathbf{9 0 0}}\left(\mathbf{P 5 3}<\mathbf{3 . 3} \times \mathbf{1 0}^{\mathbf{4}}\right)\right)\right]$} \\
\hline $\boldsymbol{t}(\mathbf{m i n})$ & \# of Samples & \# of Successes & Result & Time $(\mathbf{s})$ \\
\hline 400 & 53 & 49 & True & 597.59 \\
500 & 23 & 22 & True & 271.76 \\
600 & 22 & 22 & True & 263.79 \\
\hline
\end{tabular}

Property 1 specifies that the number of $\mathrm{p} 53$ molecules will be less than a threshold value within $t$ minutes, and it will always stay below this value during the next 900 minutes. The initial value of HMGB1 is $10^{3}$.

which informally means that the number of p53 molecules will be less than a threshold value within $t$ minutes, and it will always stay below this value during the next 900 minutes. We verified this property with various values of $t$ and the results are shown in Table 2 .

Property 2: p53's expression level increases quickly in response to various stresses, including the activation of HMGB1. We verified the property

$$
\operatorname{Pr}_{\geqslant 0.9}\left[\mathbf{F}^{100}\left(P 53>5.3 \times 10^{4}\right)\right]
$$

that is, within 100 minutes p53's level will eventually be larger than $5.3 \times 10^{4}$. SMC accepts this property as true, after sampling 38 traces (of which 37 satisfying traces).

Property 3: PI3K will be activated within a few minutes after HMGB1 binds to RAGE. We verified the following property

$$
\operatorname{Pr}_{\geqslant 0.9}\left[\mathrm{~F}^{20}\left(P I 3 K_{a} / \text { PI3K } K_{t o t}>0.5\right)\right],
$$

which means that half of PI3K will be activated within 20 minutes. We verified this property with various values of HMGB1, and the results are shown in Table 3. If HMGB1 was overexpressed $\left(10^{5}\right)$, this property was accepted as true (22 satisfying traces). But if the expression level of HMGB1 was very low, the property was rejected.

Property 4: The overexpression of HMGB1 will promote the oncoprotein CyclinE's expression before the G1-S phase transition point, thereby facilitating the G1$S$ phase transition. We verified the property

\begin{tabular}{|c|c|c|c|c|}
\hline \multicolumn{5}{|c|}{ Property 3: $\mathrm{Pr} \geq 0.9\left[\mathrm{~F}^{20}\left(P / 3 K_{a} / P I 3 K_{\text {tot }}>0.5\right)\right]$} \\
\hline HMGB1 & \# of Samples & \# of Successes & Result & Time (s) \\
\hline $10^{3}$ & 9 & 0 & False & 6.49 \\
\hline $9 \times 10^{3}$ & 380 & 315 & False & 285.16 \\
\hline $10^{5}$ & 22 & 22 & True & 16.39 \\
\hline
\end{tabular}

Table 3 Verification of property 3

Property 3 specifies that half of the PI3K molecules will be activated within 20 minutes when HMGB1 is overexpressed.

$$
\operatorname{Pr}_{\geqslant 0.9}\left[\mathrm{~F}^{600}(\text { CyclinE }>900)\right]
$$

that is, the number of CyclinE molecules will eventually exceed 900 within 600 minutes (10 hours). We verified this property with various values of HMGB1 and the results are shown in Table 4.

Property 5: Mutation in K-RAS leads to continuous activation of downstream pathways and overexpression of CyclinD in the G1 phase during HMGB1-activated signaling transduction. We verified the property:

$$
\operatorname{Pr}_{\geqslant 0.9}\left[\mathrm{~F}^{600}(\text { CyclinD > 900)], }\right.
$$

with different RAS deactivation rates $\left(d_{R A S}\right)$. The results are presented in Table 4 . Properties 4 and 5 show that the overexpression of HMGB1 and mutation of RAS (small $d_{R A S}$ value) will accelerate the expression of cell regulatory protein CyclinD/E to promote cell proliferation. However, inhibition of HMGB1 and an increase of RAS deactivation rate will prevent tumor growth.

Property 6: Within 300 minutes, CyclinE's expression level becomes very low until $50 \%$ of RAS has been activated by HMGB1. We verified the property:

$$
\operatorname{Pr}_{\geqslant 0.9}\left[(C y c l i n E<10) \mathbf{U}^{300}\left(R_{A} S_{a} / R A S_{t o t}>0.5\right)\right] .
$$

SMC accepted this property as true (22 satisfying traces).

Property 7: HMGB1 could influence the tumor suppressor protein p53's expression level, especially the first peak of p53's concentration in the G1 phase. We verified the following property:

$$
\operatorname{Pr}_{\geqslant 0.9}\left[\mathrm{~F}^{100}\left(\frac{p 53}{10000} \geqslant a \wedge \mathrm{F}^{100}\left(\frac{p 53}{10000} \leqslant 0.4\right)\right)\right],
$$

which informally means that the number of p53 protein molecules in the nucleus will eventually be greater than a threshold value $a \times 10^{4}$ within 100 minutes, after which it reduces to a low level within the next $100 \mathrm{~min}$ utes. We verified this property with various values of $a$ and HMGB1, and the results are shown in Table 5.

\section{Conclusions}

We have presented a reaction network model of the signaling transduction initiated by HMGB1. The model incorporates the contributions from the most important known signaling components of the HMGB1 signal transduction network. The model is expressed in the form of BioNetGen rules, and simulated using ODEs and Gillespie's algorithm under a range of conditions. We used Statistical Model Checking to automatically 
Table 4 Verification of property 4 and 5

\begin{tabular}{|c|c|c|c|c|c|c|c|}
\hline \multirow[b]{2}{*}{ HMGB1 } & \multicolumn{3}{|c|}{ Property 4: $\operatorname{Pr}_{\geq 0.9}\left[\mathrm{~F}^{600}(\right.$ CyclinE $\left.>900)\right]$} & \multicolumn{4}{|c|}{ Property 5: $\operatorname{Pr} \geq 0.9\left[\mathrm{~F}^{600}(\right.$ CyclinD $\left.>900)\right]$} \\
\hline & \# of Samples & \# of Success & Result & $d R A S$ & \# of Samples & \# of Success & Result \\
\hline$\overline{10^{2}}$ & 9 & 0 & False & $10^{-6}$ & 22 & 22 & True \\
\hline $10^{3}$ & 55 & 16 & False & $10^{-2}$ & 26 & 5 & False \\
\hline $10^{6}$ & 22 & 22 & True & $10^{-1}$ & 9 & 0 & False \\
\hline
\end{tabular}

Property 4 specifies that the overexpression of HMGB1 will induce the expression of cell regulatory protein CyclinE.

Property 5 specifies that the mutation of RAS will lead to the upregulation of CyclinD.

validate our model with respect to known experimental results.

Our simulations demonstrate a dose-dependent p53 and CyclinE response curve to increasing HMGB1 stimulus. This hypothesis could be tested by future experiments. In particular, overexpression of HMGB1 promotes the cell cycle regulatory proteins E2F and CyclinD/E and inhibits the pro-apoptotic protein $\mathrm{p} 53$, leading to increased cancer cell survival and decreased apoptosis. This is consistent with experimental observations in recent studies of cancer cells [11]. We also investigated the roles of different components in the pathway and predicted their activity in response to various conditions. We investigated how mutations of the RAS, ARF and P21 proteins influence the fate of the cancer cell. In particular, parameter variation showed that the mutated RAS increases the expression level of CyclinE, leading to cancer cell proliferation. Mutation or loss of the ARF protein leads to high MDM2 activity and loss of p53 expression in the face of HMGB1 overexpression, resulting in decreased apoptosis. Our model shows that the inhibition (or deactivation) of RAS, Cyclin, and Cyclin-dependent kinases (CDK) might inhibit tumor growth.

Since our proposed model is based on just three signaling pathways, we are far from capturing the entire HMGB1 network dynamics. Studies have found that HMGB1 can not only activate the PI3K-AKT and RASERK pathways, but can also activate the NF $\kappa \mathrm{B}$ signaling pathway [27], which regulates many pro-apoptotic and anti-apoptotic proteins' transcription [60]. Since HMGB1 could be released passively during necrosis,

Table 5 Verification of property 7

\begin{tabular}{llllll}
\hline Property $7: \boldsymbol{P r}_{\mathbf{0} .9}\left[\mathrm{~F}^{\mathbf{1 0 0}}\left(\boldsymbol{P} \mathbf{5 3 / 1 0 0 0 0} \geq \boldsymbol{a} \wedge \mathbf{F}^{\mathbf{1 0 0}}(\boldsymbol{P} \mathbf{5 3 / 1 0 0 0 0} \leq \mathbf{0 . 4})\right)\right]$ \\
\hline HMGB1 & $\boldsymbol{a}$ & \# of Samples & \# of Successes & Result & Time $(\mathbf{s})$ \\
\hline $10^{3}$ & 5.0 & 22 & 22 & True & 19.74 \\
$10^{3}$ & 5.5 & 20 & 3 & False & 29.02 \\
$10^{2}$ & 5.5 & 22 & 22 & True & 19.65 \\
$10^{2}$ & 6.0 & 45 & 12 & False & 56.27 \\
10 & 6.5 & 38 & 37 & True & 41.50 \\
\hline
\end{tabular}

Property 7 specifies that the increase of HMGB1's expression level can increase the first peak of p53's concentration in the G1 phase. there might exist crosstalk between the tumor necrosis factor (TNF) pathway and the HMGB1 pathway. Besides the incorporation of new pathways, recent work has demonstrated that HMGB1 can bind to p53 directly to influence p53-mediated transcriptional activity [61]. A larger network for HMGB1 signal transduction will be explored in our future work.

It has been recently observed that pancreatic tumor cells increase autophagy [11] and release HMGB1 [10] in response to chemotherapy, radiation, and hypoxia, which may promote tumor cell survival. It has been hypothesized that direct inhibition of autophagy may be another way to inhibit tumor growth and enhance the efficacy of cancer therapies [11]. The incorporation of autophagic proteins into the HMGB1 signaling pathway is worth considering in future work.

Although our current model can only qualitatively compare with the experimental behavior, it still provides valuable information about the behavior of HMGB1 signal transduction in response to different stimuli. Future experiments will enable the development of more realistic models. We anticipate that the application of model checking techniques, such as those explored in this work, will facilitate the development of targeted and effective anti-cancer therapies.

\section{Additional material}

Additional file 1: Ordinary differential equations and model parameters. The PDF file contains all the ordinary differential equations that describe the HMGB1 signal transduction model, the input parameters and their descriptions.

\section{Acknowledgements}

This work was supported by a grant from the U.S. National Science Foundation's Expeditions in Computing Program (award ID 0926181). The authors thank Michael T. Lotze (University of Pittsburgh) for calling their attention to HMGB1 and for helpful discussions of the topic. H.G. would like to thank Marco E. Bianchi (San Raffaele University) for email discussions on HMGB1. We would also like to thank llya Korsunsky and Màtè L. Nagy for their comments on this paper.

This article has been published as part of BMC Bioinformatics Volume 11 Supplement 7, 2010: Ninth International Conference on Bioinformatics (InCoB2010): Bioinformatics. The full contents of the supplement are available online at http://www.biomedcentral.com/1471-2105/11?issue=S7. 


\section{Author details}

${ }^{1}$ Computer Science Department, Carnegie Mellon University, Pittsburgh, PA 15213, USA. ${ }^{2}$ Department of Computational Biology, University of Pittsburgh, Pittsburgh, PA 15260, USA.

\section{Authors' contributions}

E.M.C., J.R.F., H.G. and P.Z. proposed the project; H.G. and P.Z. wrote the manuscript; H.G. wrote the BioNetGen code and performed the numerical simulations and formal verifications; P.Z. wrote the statistical model checker; A.K. wrote the model checker code for BioNetGen. All authors read and approved the final manuscript.

\section{Competing interests}

The authors declare that there are no competing interests.

\section{Published: 15 October 2010}

\section{References}

1. Hanahan D, Weinberg RA: The hallmarks of cancer. Cell 2000, 100:57-70.

2. Scaffidi P, Misteli T, Bianchi M: Release of chromatin protein HMGB1 by necrotic cells triggers inflammation. Nature 2002, 418:191-195.

3. Bonaldi T, Talamo F, Scaffidi P, Ferrera D, Porto A, Bachi A, Rubartelli A: Monocytic cells hyperacetylate chromatin protein HMGB1 to redirect it towards secretion. EMBO J 2003, 22:5551-5560.

4. Wang $\mathrm{H}$, Bloom $\mathrm{O}$, Zhang $\mathrm{M}$, Vishnubhakat J, Ombrellino M, Frazier A, Yang H: HMG-1 as a late mediator of endotoxin lethality in mice. Science $1999,285: 248$

5. Dumitriu IE, Baruah $P$, Valentinis $B$, et al: Release of high mobility group box 1 by dendritic cells controls $T$ cell activation via the receptor for advanced glycation end products. The Journal of Immunology 2005, 174:7506-7515.

6. Semino C, Angelini G, Poggi A, Rubartelli A: NK/iDC interaction results in IL-18 secretion by DCs at the synaptic cleft followed by NK cell activation and release of the DC maturation factor HMGB1. Blood 2005, 106:609-616.

7. Sparvero L, Asafu-Adje D, Kang R, Tang D, Amin N, et al: RAGE (Receptor for Advanced Glycation Endproducts), RAGE ligands, and their role in cancer and inflammation. Journal of Translational Medicine 2009, 7:17.

8. Ellerman JE, Brown CK, de Vera M, Zeh HJ, Billiar T, et al: Masquerader: high mobility group box-1 and cancer. Clinical Cancer Research 2007, 13:2836-2848.

9. Lotze MT, Tracey K: High-mobility group box 1 protein (HMGB1): nuclear weapon in the immune arsenal. Nature Reviews Immunology 2005, 5:331-342.

10. Vakkila J, Lotze MT: Inflammation and necrosis promote tumour growth Nature Reviews Immunology 2004, 4:641-648.

11. Kang R, Tang D, Schapiro NE, Livesey KM, Farkas A, Loughran P, Lotze MT, Zeh $\mathrm{HJ}$ : The receptor for advanced glycation end products (RAGE) sustains autophagy and limits apoptosis, promoting pancreatic tumor cell survival. Cell Death Differ 2010, 17(4):666-676.

12. Brezniceanu ML, Volp K, Bosser S, Solbach C, Lichter P, et al: HMGB1 inhibits cell death in yeast and mammalian cells and is abundantly expressed in human breast carcinoma. FASEB Journal 2003, 17:1295-1297.

13. Vogelstein B, Lane D, Levine AJ: Surfing the p53 network. Nature 2000, 408:307-310.

14. Minella AC, Grim JE, Welcker M, Clurman BE: p53 and SCFFbw7 cooperatively restrain cyclin E-associated genome instability. Oncogene 2007, 26:6948-6953.

15. Pestov DG, Strezoska A, Lau LF: Evidence of p53-dependent cross-talk between ribosome biogenesis and the cell cycle: effects of nucleolar protein Bop1 on G1/S transition. Molecular and Cellular Biology 2001, 21(13):4246-4255.

16. Wee KB, Aguda BD: Akt versus $p 53$ in a network of oncogenes and tumor suppressor genes regulating cell survival and death. Biophysical Journal 2006, 91:857-865.

17. Wee KB, Surana U, Aquda BD: Oscillations of the p53-Akt Network: Implications on Cell Survival and Death. PloS One 2009, 4:e4407.

18. Ciliberto A, Novak B, Tyson J: Steady States and Oscillations in the p53/ Mdm2 Network. Cell Cycle 2005, 4(3):488-493.
19. Zhang T, Brazhnik P, Tyson J: Exploring mechanisms of the DNA-damage response: $\mathrm{p} 53$ pulses and their possible relevance to apoptosis. Cell Cycle 2007, 6:85-e10.

20. Puszynski K, Hat B, Lipniacki T: Oscillations and bistability in the stochastic model of p53 regulation. Journal of Theoretical Biology 2008, 254:452-465.

21. Bottani $S$, Grammaticos B: Analysis of a minimal model for $p 53$ oscillations. Journal of Theoretical Biology 2007, 249:235-245.

22. Clarke EM, Grumberg O, Peled DA: Model Checking MIT Press 1999.

23. Jha SK, Clarke EM, Langmead CJ, Legay A, Platzer A, Zuliani P: A bayesian approach to model checking biological system. CMSB, Volume 5688 of LNCS 2009, 218-234.

24. Langmead CJ: Generalized Queries and Bayesian Statistical Model Checking in Dynamic Bayesian Networks: Application to Personalized Medicine. CSB 2009, 201-212.

25. Rizk A, Batt G, Fages F, Soliman S: On a continuous degree of satisfaction of temporal logic formulae with applications to systems biology. CMSB, Volume 5307 of LNCS 2008, 251-268.

26. Larris S, Levine AJ: The p53 pathway: positive and negative feedback loops. Oncogene 2005, 24:2899-2908.

27. van Beijnum JR, Buurman WA, Griffioen AW: Convergence and amplification of toll-like receptor (TLR) and receptor for advanced glycation end products (RAGE) signaling pathways via high mobility group B1. Angiogenesis 2008, 11:91-99.

28. Barak $Y$, Juven T, Haffiner $R$, Oren $M: m d m 2$ expression is induced by wild type p53 activity. EMBO J 1993, 12:461-468.

29. Haupt Y, Maya R, Kasaz A, Oren M: Mdm2 promotes the rapid degradation of p53. Nature 1997, 387:296-299.

30. Blanco-Aparicio C, Renner O, Leal J, Carnero A: PTEN, more than the AKT pathway. Carcinogenesis 2007, 28:1379-1386.

31. Downward J: Targeting RAS signalling pathways in cancer therapy. Nature Reviews Cancer 2003, 3:11-22

32. Yao G, Lee TJ, Mori S, Nevins J, You L: A bistable Rb-E2F switch underlies the restriction point. Nature Cell Biology 2008, 10:476-482.

33. Sherr CJ, McCormick F: The RB and p53 pathways in cancer. Cancer Cell 2002, 2:103-112.

34. Sears R, Nevins J: Signaling networks that link cell proliferation and cell fate. The Journal of Biological Chemistry 2002, 277:11617-11620.

35. Nevins JR: The Rb/E2F pathway and cancer. Human Molecular Genetics 2001, 10:699-703.

36. Bardeesy N, DePinho RA: Pancreatic cancer biology and genetics. Nature Reviews Cancer 2002, 2(12):897-909.

37. Mao J, Perez-losada J, Wu D, DelRosario R, Tsunematsu R, Nakayama KI, Brown K, Bryson S, Balmain A: Fbxw7/Cdc4 is a p53-dependent, haploinsufficient tumour suppressor gene. Nature 2004, 432:775-779.

38. Hlavacek WS, Faeder JR, Blinov ML, Posner RG, Hucka M, Fontana W: Rules for modeling signal-transduction system. Sci STKE 2006, 2006(344):re6, re6.

39. Bolouri H: Computational Modeling of Gene Regulatory Networks: a primer Imperial College Press 2008.

40. Yang $\mathrm{H}, \mathrm{Hsu} \mathrm{C}$, Hwang M: An Analytical Rate Expression for the Kinetics of Gene Transcription Mediated by Dimeric Transcription Factors. Journal of Biochemistry 2007, 142:135-144.

41. Alon U: An introduction to systems biology: design principles of biological circuits Chapman \& Hall 2007.

42. Gillespie DT: A general method for numerically simulating the stochastic time evolution of coupled chemical reactions. Journal of Computational Physics 1976, 22(4):403-434

43. Gong H, Sengupta H, Linstedt A, Schwartz R: Simulated De Novo Assembly of Golgi Compartments by Selective Cargo Capture during Vesicle Budding and Targeted Vesicle Fusion. Biophysical Journal 2008, 95:1674-1688.

44. Gong H, Guo Y, Linstedt A, Schwartz R: Discrete, continuous, and stochastic models of protein sorting in the Golgi apparatus. Phys Rev $E$ Stat Nonlin Soft Matter Phys 2010, 81:011914.

45. Lipniacki T, Hat T, Faeder JR, Hlavacek WS: Stochastic effects and bistability in T cell receptor signaling. Journal of Theoretical Biology 2008, 254:110-122.

46. HMGB1 BioNetGen Code. [http://www.cs.cmu.edu/ haijung/research/ HMGB1model.bngl].

47. Clarke EM, Emerson EA, Sifakis J: Model checking: algorithmic verification and debugging. Commun ACM 2009, 52(11):74-84. 
48. Pnueli A: The Temporal Logic of Programs. FOCS 1977, 46-57.

49. Zuliani P, Platzer A, Clarke EM: Bayesian statistical model checking with application to Simulink/Stateflow verification. HSCC 2010, 243-252.

50. Younes HLS, Simmons RG: Probabilistic Verification of Discrete Event Systems Using Acceptance Sampling. CAV, Volume 2404 of LNCS 2002, 223-235.

51. Younes HLS, Simmons RG: Statistical probabilistic model checking with a focus on time-bounded properties. Information and Computation 2006, 204(9):1368-1409.

52. Geva-Zatorsky N, Rosenfeld N, Itzkovitz S, Milo R, Sigal A, Dekel E, Yarnitzky T, Liron Y, Polak P, Lahav G, Alon U: Oscillations and variability in the p53 system. Molecular Systems Biology 2006, 2:2006.0033.

53. Hahn WC, Weinberg RA: Modelling the molecular circuitry of cance. Nature Reviews Cancer 2002, 2:331-341.

54. Altomare D, Wang H, Skele K, Rienzo AD, Klein-Szanto A, Godwin A, Testa J: AKT and mTOR phosphorylation is frequently detected in ovarian cancer and can be targeted to disrupt ovarian tumor cell growth. Oncogene 2004, 23:5853-7.

55. Rhodes N, Heerding D, Duckett D, et al: Characterization of an Akt kinase inhibitor with potent pharmacodynamic and antitumor activity. Cancer Research 2008, 68:2366.

56. van Krieken J, et al: KRAS mutation testing for predicting response to anti-EGFR therapy for colorectal carcinoma: proposal for an European quality assurance program. Virchows Arch 2008, 453:417-431.

57. Mclnnes C: Progress in the evaluation of CDK inhibitors as anti-tumor agents. Drug Discovery Today 2008, 13(1920):875-881

58. Malumbres $M$, Pevarello $P$, Barbacid M, Bischoff J: CDK inhibitors in cancer therapy: what is next? Trends in Pharmacological Sciences 2008, 29:16

59. Rotblat B, Ehrlich M, Haklai R, Kloog Y: The Ras inhibitor farnesylthiosalicylic acid (Salirasib) disrupts the spatiotemporal localization of active Ras: a potential treatment for cancer. Methods in Enzymology 2008, 439:467-489.

60. Lipniacki T, Paszek P, Brasier A, Luxon B, Kimmel M: Crosstalk between p53 and nuclear factor-kB systems: pro-and anti-apoptotic functions of NFkB. Journal of Theoretical Biology 2004, 228:195-215.

61. Imamura T, Izumi H, Nagatani G, Ise T, Nomoto M, Iwamoto Y, Kohno K Interaction with $\mathrm{p} 53$ enhances binding of cisplatin-modified DNA by high mobility group 1 protein. J Biol Chem 2001, 276:7534-7540.

doi:10.1186/1471-2105-11-S7-S10

Cite this article as: Gong et al: Analysis and verification of the HMGB1 signaling pathway. BMC Bioinformatics 2010 11(Suppl 7):S10.

\section{Submit your next manuscript to BioMed Central and take full advantage of:}

- Convenient online submission

- Thorough peer review

- No space constraints or color figure charges

- Immediate publication on acceptance

- Inclusion in PubMed, CAS, Scopus and Google Scholar

- Research which is freely available for redistribution

Submit your manuscript at www.biomedcentral.com/submit 